\title{
NT-probnp Variability as a Predictor of Adverse Outcomes in Patients With Cardiorenal Syndrome Type 2
}

\section{Mingming Ma}

Jinan University First Affiliated Hospital https://orcid.org/0000-0003-2474-2415

\section{Xiangnan Dong}

Jinan University First Affiliated Hospital

Shuang Cui

Jinan University First Affiliated Hospital

\section{Berthold Hocher}

University Medical Centre Mannheim

\section{Shufei Zeng}

Jinan University First Affiliated Hospital

\section{Wenxue Liang}

Jinan University First Affiliated Hospital

\section{Qiang Li}

Dongguan Hospital of Traditional Chinese Medicine

\section{Xiaoyi Chen}

Jinan University First Affiliated Hospital

\section{Xin Chen}

Jinan University First Affiliated Hospital

\section{Yu Meng}

Jinan University First Affiliated Hospital

\section{Yongping Lu}

Jinan University First Affiliated Hospital

\section{Lianghong Yin ( $\square$ jnufu09@126.com )}

Institute of Nephrology and Blood Purification, The First Affiliated Hospital of Jinan University, Jinan University, Guangzhou, China. https://orcid.org/0000-0002-7306-8570

\section{Research article}

Keywords: NT-proBNP variability, cardiorenal syndrome, in-hospital outcomes 
DOl: https://doi.org/10.21203/rs.3.rs-106286/v1

License: (c) (1) This work is licensed under a Creative Commons Attribution 4.0 International License. Read Full License 
NT-proBNP variability as a predictor of adverse outcomes in patients with cardiorenal syndrome type 2

Mingming Ma ${ }^{1}$, Xiangnan Dong ${ }^{1}$, Shuang Cui ${ }^{1}$, Berthold Hocher $^{2}$, Shufei Zeng ${ }^{1}$, Wenxue Liang ${ }^{1}$,Qiang $\mathrm{Li}^{3}$, Xiaoyi Chen ${ }^{1}$, Xin Chen ${ }^{1}$, Yu Meng ${ }^{1}$, Yongping $\mathrm{Lu}^{1}$, Lianghong Yin $^{1 *}$

Mingming Ma and Xiangnan Dong contributed equally to this work.

${ }^{1}$ Institute of Nephrology and Blood Purification, The First Affiliated Hospital of Jinan University, Jinan University, Guangzhou, China.

${ }^{2}$ Fifth Department of Medicine (Nephrology/Endocrinology/Rheumatology), University Medical Centre Mannheim, University of Heidelberg, Heidelberg, Germany.

${ }^{3}$ Department of Nephrology, Dongguan Hospital of traditional Chinese Medicine,Dongguan,China.

\section{*Corresponding author:}

Prof. Lianghong Yin, MD

Institute of Nephrology and Blood Purification, The First Affiliated Hospital of Jinan 
University, Jinan University, Guangzhou 510632, China

No.613, West Huangpu Avenue, Guangzhou 510632, China

Fax: +8602038688310

Tel: +862038688449

E-mail address: jnufu09@126.com

Key words: NT-proBNP variability, cardiorenal syndrome, in-hospital outcomes

\title{
Word count: 4,638
}

\begin{abstract}
Background: N-terminal prohormone B-type natriuretic peptide (NT-proBNP) is a significant predictor of cardiovascular and all-cause mortality in patients with chronic heart failure (HF) without chronic kidney disease (CKD). However, it is unknown whether variability of NT-proBNP is predictive of the outcomes of those with advanced HF and CKD.
\end{abstract}

Methods: Intra-individual fluctuations in NT-proBNP values from baseline to follow-up were determined to assess the prognostic value of NT-proBNP variability in patients with cardiorenal syndrome (CRS) type 2 in a retrospective cohort of 136 patients treated for advanced HF with CKD at the First Affiliated Hospital of Jinan University from 2012 to 2016. Patients hospitalized for CRS type 2 who underwent follow-up examinations were eligible for study inclusion and grouped according to 
optimal cutoff values of receiver operating characteristic (ROC) curve analysis of $\log 10$ (NT-proBNP) variability. The primary outcomes were major adverse renal and cardiac events( a composite of acute kidney disease, nonfatal myocardial infarction, cardiac death, stroke or maintenance hemodialysis). Secondary outcomes were repeated hospitalization for heart failure or all cause death.

\section{Results:}

The results showed that higher NT-proBNP variability group was associated with lower hemoglobin(p $<0.01)$ and plasma serum albumin levels(p $<0.02)$, and greater decline in left ventricular ejection fraction $(\mathrm{p}<0.02)$ compared with the lower NT-proBNP variability group. At a median follow-up of 22 (interquartile range, 5-32) months, Individuals in the higher NT-proBNP variability group was associated with a greater risk of major adverse renal and cardiac events $(\mathrm{HR}=3.467,95 \% \mathrm{CI}=$ $1.463-8.215, p=0.01)$. ROC analysis revealed that NT-proBNP variability rather than average NT-proBNP level, was a better predictive biomarker of the primary outcomes $(\mathrm{AUC}=0.726,95 \% \mathrm{CI}=0.640-0.812)$ and secondary outcomes $(\mathrm{AUC}=0.701,95 \%$ $\mathrm{CI}=0.611-0.791)$. Kaplan-Meier analysis showed that NT-proBNP variability of $\geq 0.272$ was significantly correlated with a higher prevalence of primary and secondary outcomes $(p<0.03)$. This correlation remained significant in multivariate Cox regression models after adjustments for age, anemia, and traditional risk factors.

Conclusions: NT-proBNP variability might serve as an independent predictor for major adverse renal and cardiac events and all-cause death in patients with CRS type 2. 


\section{Introduction}

Heart failure (HF) is a complex syndrome with typical symptoms and specific signs caused by structural and/or functional cardiac abnormalities [1]. HF remains a major and increasing public health concern worldwide because of the relatively high morbidity and mortality rates [1].

HF and renal dysfunction are frequently concomitant in hospitalized patients and interact to worsen prognosis and significantly increase the risks of prolonged hospitalization and even death [2, 3]. Five subtypes of cardiorenal syndrome (CRS) have been proposed to describe the complex pathophysiological disorders of both the heart and kidney [4]. The occurrence or progression of chronic kidney disease (CKD) in the context of a primary chronic abnormality in cardiac function is termed CRS type $2[4]$.

Despite the widespread recognition of the importance of CRS type 2, progress toward an understanding of this syndrome has remained challenging [5]. Biomarkers that provide insights into both cardiac and renal disorders have the potential to reveal some of the intricacies involved in assessing heart-kidney interactions and to facilitate overcoming these challenges.

Natriuretic peptides, including B-type natriuretic peptide (BNP) and N-terminal prohormone B-type natriuretic peptide (NT-proBNP), are the most established diagnostic and prognostic biomarkers of CRS type 2, and have been reported to be strong and independent predictors of the morbidity and mortality of patients with various cardiovascular diseases, especially HF and coronary heart disease [6-11]. 
Notably, NT-proBNP is predominantly synthesized and released constitutively from ventricular cardiac myocytes in response to increased mechanical stretching and elevated filling pressures, and appears to be adversely affected by severe deterioration of renal function [12]. The plasma NT-proBNP concentration reflects aspects of cardiac and renal dysfunction, and has been identified as an independent prognostic predictor of CHF patients with CKD $[13,14]$, as independently predicted by estimated glomerular filtration rate (eGFR), age, New York Heart Association functional class, and ejection fraction [13]. However, it is well-known that NT-proBNP has a considerable intra-individual biological variability and disease progression may result in substantial changes in NT-proBNP levels over time [7, 15-17]. Hence, measurements of NT-proBNP variability could offer additional prognostic information.

Therefore, the aim of the present was to determine whether the dynamic changes in NT-proBNP levels could serve as a biomarker of risk stratification and prognosis of patients with CRS type 2 .

\section{Methods}

\section{Study population and data collection}

The study cohort consisted of 136 patients diagnosed with CRS type 2 at the Department of Cardiovascular of the First Affiliated Hospital of Jinan University between 2012 and 2016. The 2019 American Heart Association definition of CRS type 2 was used as the major screening criteria. The diagnosis of patients with 
suspected CRS type 2 was confirmed on a case-by-case basis by reference to the hospital electronic medical record system. Patients with stage $5 \mathrm{CKD}$ requiring chronic dialysis, kidney transplantation, HF following cardiac surgery, and/or multi-organ failure were excluded from analysis. Patients with no records of plasma NT-proBNP concentrations before admission or during hospitalization were also excluded.The selection of study participants is described in Fig. 1.

A structured medical history was taken for each patient. The following data were extracted from the hospital database: age, sex, body height and weight, heart rate, systolic and diastolic blood pressure (SBP and DBP, respectively), previous history of hypertension, cerebrovascular disease, chronic obstructive pulmonary disease (COPD), liver disease, atrial fibrillation (AF), diabetes mellitus, hospitalization due to myocardial infarction, maintenance hemodialysis, and all-cause mortality.

The mean values of three measurements of SBP and DBP were used for analysis. The diagnosis of chronic HF was based on medical history, symptoms, physical examination findings, imaging studies, and biomarkers (NT-proBNP). Medical history included coronary heart disease and hypertension. Symptoms and clinical findings included dyspnea, fatigue, edema, chest pain, persistent cough or wheezing with pink blood-tinged phlegm, elevated jugular venous pressure, and wet rales in the lung. Chest X-rays were used to assess the degree of pulmonary congestion and to exclude pneumonia and other causes of symptoms. Echocardiography was performed to measure the ejection fraction for classification of HF. A plasma NT-proBNP 
concentration of $>300 \mathrm{pg} / \mathrm{mL}$ was considered elevated. CKD was defined as an eGFR range of 15 to $60 \mathrm{~mL} / \mathrm{min} / 1.73 \mathrm{~m}^{2}$.

Patients were followed monthly. NT-proBNP levels were obtained from laboratory test reports during hospitalization and at least two post-baseline measurements were included for analysis. Participants were assigned to one of two groups based on $\log 10$ (NT-proBNP) variability: (1) low $\log 10$ (NT-proBNP) variability $(<0.27)$ or $(2)$ high $\log 10$ (NT-proBNP) variability $(\geq 0.27)$. The primary outcomes were major adverse renal and cardiac events( a composite of acute kidney disease, nonfatal myocardial infarction, cardiac death ,stroke or maintenance hemodialysis). Secondary outcomes were repeated hospitalization for heart failure or all cause death.

\section{NT-proBNP variability}

The NT-proBNP levels was logarithmically transformed to meet the multinormality assumption. NT-proBNP variability was defined as average absolute difference between each $\log 10$ ( NT-proBNP) and the mean during the period of hospitalization.

Laboratory parameters (i.e., hemoglobin, serum albumin, serum creatinine, and NT-proBNP) were measured in the Central Clinical Laboratory of the First Affiliated Hospital of Jinan University. All measurements were subject to routine quality controls in accordance with the laws of the People's Republic of China. eGFR was estimated using the Modification of Diet in Renal Disease Study equation as follows: $\mathrm{eGFR}=175 \times(\text { serum creatinine })^{-1.154} \times(\text { age })^{-0.203} \times 0.742($ female $)$. 


\section{Statistical analysis}

Continuous variables are expressed as the mean \pm standard deviation or the median and interquartile ranges, while categorical variables are presented as percentages or frequencies. NT-proBNP levels are presented as categorical variables or as continuous variables after natural logarithmic transformation. For univariate analysis, normally distributed values were compared between the two groups using the Student's $t$-test, while non-normally distributed values were compared using the Mann-Whitney test. The Pearson $\chi^{2}$ test and Kruskal-Wallis test were applied for analyses of nominal and ordinal variables, respectively. Primary outcome-free survival curves were constructed with the Kaplan-Meier method and differences between the two groups were evaluated with the log-rank test. The associations between NT-proBNP variability and the primary endpoints were evaluated using the multivariate Cox proportional hazard regression model adjusted for baseline characteristics. Although a probability $(p)$ value of $<0.05$ was considered significant for all inferential statistics, a $p$ value threshold of $\leq 0.2$ was used for univariate analysis for inclusion of putative risk factors into the multivariate model. All data analyses were performed using IBM SPSS Statistics for Windows, version 19.0. (IBM Corporation, Armonk, NY, USA).

\section{Results}

\section{Demographic characteristics}

Of the 136 patients, $67(49.3 \%)$ and $69(50.7 \%)$ were assigned to the low and high NT-proBNP variability groups, respectively. The baseline characteristics of the 
patients across the two groups are shown in Table 1. The distributions of the $\log 10(\mathrm{NT}-\mathrm{proBNP})$ and $\log 10(\mathrm{NT}-\mathrm{proBNP})$ variability values are presented in Fig. 2. The mean baseline $\log 10(\mathrm{NT}$-proBNP) value of the patients was $3.48 \pm 0.59$. The median time between the first and last measurements of NT-proBNP was 22 months. The median number of NT-proBNP assay measurements was 5 (range, 3-32). The median $\log 10$ (NT-proBNP) variability value (as measured by average successive variability) was 0.281 . The mean $\log _{10}(\mathrm{NT}$-proBNP) variability value of the patients was $0.28 \pm 0.12$. Statistically, there were no significant differences between the two groups in terms of age, sex, body mass index, serum creatinine, SBP, DBP, eGFR, NT-proBNP, hypertension, cerebrovascular disease, diabetes, COPD, liver disease, AF, New York Heart Association (NYHA) classification $\geq$ III, and the use of angiotensin-converting enzyme inhibitors, angiotensin II receptor blockers, or percutaneous coronary intervention (PCI). The baseline concentrations of hemoglobin and albumin were significantly lower in patients with a NT-proBNP variability value of $\geq 0.27$ ( $\mathrm{p}=0.01$ and $<0.02$, respectively). Correspondingly, patients with high NT-proBNP variability had a lower left ventricular ejection fraction $(p=0.02)$.

\section{Correlations between NT-proBNP variability and the outcomes}

Over a median follow-up period of 22 (interquartile range $=14-36$ ) months, there were50 (36.8\%) major adverse renal and cardiac events occurred and 60 cases of incident repeated hospitalization for heart failure or all cause death. The incidence of the primary outcomes significantly increased in the higher NT-proBNP variability 
group compared with lower group as shown in table 2.

The predictive value of NT-proBNP variability in regards to the primary and secondary endpoint of this study was calculated by the area under the receiver operating characteristic curve (ROC-AUC), as shown in Fig3,5. Compared with average NT-proBNP level, NT-proBNP variability had reasonable accuracy as a predictor of the primary outcomes $(\mathrm{AUC}=0.726 ; 95 \% \mathrm{CI}=0.640-0.812)$ and secondary outcomes $(\mathrm{AUC}=0.701 ; 95 \% \mathrm{CI}=0.611-0.791)$.

Kaplan-Meier analysis was used to assess differences in the occurrence of the primary and secondary outcomes until the follow-up based on NT-proBNP variability values of $<0.272$ and $\geq 0.272$. A NT-proBNP variability value of $\geq 0.272$ was significantly associated with a higher prevalence of the major adverse renal and cardiac events and secondary endpoints until the follow-up (log-rank Mantel-Cox test; $p<0.03$, see Fig 4,6).

Multivariate Cox regression models were used to identify independent associations between NT-proBNP variability and elevated risks for the primary outcomes (Table 3). Model A used age, anemia, hypertension, diabetes mellitus, left ventricular ejection fraction (LVEF), hypoproteinemia, and baseline eGFR and PCI as covariates $\left(\chi^{2}=25.3, p=0.001\right)$. The addition of $\log _{10}(\mathrm{NT}-\mathrm{proBNP})$ variability as a continuous variable to the same covariates as used in model A improved the overall strength of model B $\left(\chi^{2}=29.2, p=0.001\right)$. In model C, NT-proBNP variability was applied as a categorical variable to the same covariates as used in model A (NT-proBNP variability $\geq 0.27 \mathrm{OR}<0.27$ ), which resulted in a similar model strength 
as Model B $\left(\chi^{2}=27.1, p=0.001\right)$.

\section{Discussion}

The key finding of this study include the following: first, higher fluctuations in NT-proBNP levels of patients with CRS type 2 was associated with greater risks for the major adverse outcomes of acute kidney disease, nonfatal myocardial infarction, cardiac death ,stroke ,maintenance hemodialysis, repeated hospitalization for heart failure and all cause death. second, ROC analysis demonstrated that NT-proBNP variability, rather than average NT-proBNP level,was highly predictive of the major adverse renal and cardiac events, repeated hospitalization for heart failure or all cause death, with an optimal cutoff of $\geq 0.272$. The results of Kaplan-Meier analysis found that patients with NT-proBNP variability of $\geq 0.272$ were at a significantly greater risk of the adverse outcomes. Multivariate Cox regression models demonstrated that this correlation was independent from other important risk factors. Taken together, these results confirmed independent associations between NT-proBNP variability and the occurrence of major adverse renal and cardiac events, repeated hospitalization for heart failure and all cause death.

Previous studies have demonstrated that NT-proBNP can be used as a biomarker for the diagnosis, prognosis, risk stratification, and therapeutic effect assessment of patients with $\mathrm{HF}[18,19]$. In patients without CKD, NT-proBNP is a major independent prognostic factor for HF. In addition, several studies that investigated the relationship between circulating NT-proBNP concentrations and mortality risk 
reported that for every $500-\mathrm{ng} / \mathrm{mL}$ increase in baseline NT-proBNP levels, there is a corresponding $3.8 \%$ relative increase in the risk of death after adjustment for other traditional risk factors [20]. Among patients with baseline NT-proBNP levels greater than the median value, there was a two-fold increase in the risk of cardiovascular and all-cause mortality as compared with those with baseline levels less than the median value [21]. A dramatic decrease in NT-proBNP levels by at least $30 \%$ has been shown to significantly decrease the risks of hospital readmission and death [22]. As such, NT-proBNP-guided treatment of HF is an important intervention. The results of the Christchurch New Zealand pilot trial suggested that NT-proBNP-guided treatment of HF reduced the incidence of total cardiovascular events (death, hospitalization, and HF decompensation) and delayed the time to a first event as compared with intensive clinically guided therapy [23]. However, larger trials, such as the STARBRITE, TIME-CHF, and PRIMA studies, failed to show a clear advantage of NT-BNP-guided therapy [24-26]. The generalizability of these trial results has, however, been called into question because of the usual exclusion of patients with renal failure. The results of the present study showed that a NT-proBNP variability value of $\geq 0.272$ was a strong and independent risk factor for the adverse outcomes after adjusting for age, anemia, hypertension, diabetes mellitus, LVEF, hypoproteinemia, and baseline eGFR and PCI, in accordance with the findings of previous studies. Hence, the results of the current study may have relevant clinical implications. Calculation of a threshold using the NT-proBNP variability ROC curve for adverse outcomes resulted in a cut-off value for NT-proBNP variability of $\geq 0.272$ to identify patients at risk for CHF with 
CKD, which might be extremely important information for clinical management.

Although maintaining stable NT-proBNP levels over time is recommended, the usual pattern is that NT-proBNP concentrations decrease and then increase. However, it remains controversial whether such fluctuations affect the prognosis of CHF with CKD. Here, a review of the data of 136 patients with CRS type 2 found that high NT-proBNP variability was associated with a significant increase in the risk of major adverse renal and cardiac events, repeated hospitalization for heart failure and all cause death.

The results of this study showed that NT-proBNP variability might serve as a pre-interventional highly predictive biomarker for adverse outcomes (acute kidney disease, nonfatal myocardial infarction, cardiac death, stroke or maintenance hemodialysis). Patients at high risk for adverse outcomes based on NT-proBNP variability before intervention may need a much closer follow-up, as compared to low risk patients. Repeated NT-proBNP measurements seem to be very meaningful for CRS type 2 patients, thus clinicians may need to pay more attention to such patients with high NT-proBNP variability.

There may be potential limitations to this study when interpreting these findings. First, this study was based on data from a single center and the sample size was relatively small. Hence, further studies of multicenter data are needed to enhance the validity of these results. Second, this study was retrospective and the reasons for the dropping-out of some patients were missing, which could lead to biased results. Third, other biomarkers, such as serum troponins and cystatin $\mathrm{C}$, which are considered to 
predict outcomes, were not assessed in this study. Therefore, future studies should also include more traditional biomarkers.

In conclusion, NT-proBNP variability was an important prognostic marker of patients with CRS type 2. High NT-proBNP variability was strongly predictive of a worse outcome and provided an independent prognostic value beyond that of relevant risk factors, including eGFR. However, further investigations are needed to validate the additional benefits of evaluating NT-proBNP variability for the prediction of long-term adverse outcomes in patients with CRS type 2.

\section{Acknowledgements}

We thank Ting Luo for her assistance with formatting of this manuscript.

\section{Study approval}

The protocol of this retrospective study was approved by the Institutional Ethical Review Board of Jinan University (Guangzhou, China) and conducted in accordance with the tenets outlined in the Declaration of Helsinki.

\section{Funding}

This study was supported by the Guangdong Academician Workstation of Industry-University-Research Cooperation (grant no. 2013B090400004).

\section{Conflict of Interest Statement}


All authors declared no competing interests.

\section{Author Contributions Statement}

Mingming Ma and Xiangnan Dong generated the data for the manuscript and partially wrote the manuscript. Xiaoyi Chen and Xin Chen generated the data for the manuscript. Mingming Ma,and Lianghong Yin discussed the results of the manuscript. Shuang Cui and Yongping Lu edited the manuscript. Berthold Hocher, Shufei Zeng and Wenxue Liang performed statistical analyses in the manuscript. Berthold Hocher, Yu Meng ,and Lianghong Yin are the guarantors of this work and, as such, had full access to all the data in the study and take responsibility for the integrity of the data and the accuracy of the data analysis.

\section{References}

1. Ponikowski P, Voors AA, Anker SD, et al: 2016 ESC Guidelines for the diagnosis and treatment of acute and chronic heart failure: The Task Force for the Diagnosis and Treatment of Acute and Chronic Heart Failure of the European Society of Cardiology (ESC) Developed with the special contribution of the Heart Failure Association (HFA) of the ESC. Eur Heart J 2016, 37(27):2129-2200.

2. Damman K, Valente MA, Voors AA, et al : Renal impairment, worsening renal function, and outcome in patients with heart failure: an updated meta-analysis. Eur Heart J 2014, 35(7):455-469.

3. Filippatos G, Farmakis D, Parissis J: Renal dysfunction and heart failure: things are seldom what they seem. Eur Heart J 2014, 35(7):416-418. 
4. Shah BN, Greaves K: The cardiorenal syndrome: a review. Int J Nephrol 2010, 2011:920195.

5. Cruz DN, Schmidt-Ott KM, Vescovo G, et al : Pathophysiology of cardiorenal syndrome type 2 in stable chronic heart failure: workgroup statements from the eleventh consensus conference of the Acute Dialysis Quality Initiative (ADQI). Contrib Nephrol 2013, 182:117-136.

6. Komajda M, Carson PE, Hetzel S, et al: Factors associated with outcome in heart failure with preserved ejection fraction: findings from the Irbesartan in Heart Failure with Preserved Ejection Fraction Study (I-PRESERVE). Circ Heart Fail 2011, $4(1): 27-35$.

7. Zile MR, Claggett BL, Prescott MF, et al: Prognostic implications of changes in N-terminal Pro-B-Type natriuretic peptide in patients with heart failure. $\mathrm{J} \mathrm{Am} \mathrm{Coll}$ Cardiol 2016, 68(22):2425-2436.

8. Lainchbury JG, Troughton RW, Strangman KM, et al: N-terminal pro-B-type natriuretic peptide-guided treatment for chronic heart failure: results from the BATTLESCARRED (NT-proBNP-Assisted Treatment To Lessen Serial Cardiac Readmissions and Death) trial. J Am Coll Cardiol 2009, 55(1):53-60.

9. Pfisterer M, Buser P, Rickli H, et al: BNP-guided vs symptom-guided heart failure therapy: the Trial of Intensified vs Standard Medical Therapy in Elderly Patients With Congestive Heart Failure (TIME-CHF) randomized trial. JAMA 2009, 301(4):383-392.

10. Kragelund C, Gronning B, Kober L, et al: N-terminal pro-B-type natriuretic 
peptide and long-term mortality in stable coronary heart disease. N Engl J Med 2005, 352(7):666-675.

11. Maisel AS, Krishnaswamy P, Nowak RM, et al: Rapid measurement of B-type natriuretic peptide in the emergency diagnosis of heart failure. $N$ Engl J Med 2002, 347(3):161-167.

12. McCullough PA, Sandberg KR: B-type natriuretic peptide and renal disease. Heart Fail Rev 2003, 8(4):355-358.

13. Bruch C, Fischer C, Sindermann J, et al: Comparison of the prognostic usefulness of N-terminal pro-brain natriuretic peptide in patients with heart failure with versus without chronic kidney disease. Am J Cardiol 2008, 102(4):469-474.

14. Bruch C, Reinecke H, Stypmann J, et al: N-terminal pro-brain natriuretic peptide: Kidney disease and outcome in patients with chronic heart failure. $J$ Heart Lung Transplant 2006, 25(9):1135-1141.

15. Masson S, Latini R, Anand IS, et al: Prognostic value of changes in N-terminal pro-brain natriuretic peptide in Val-HeFT (Valsartan Heart Failure Trial). $J$ Am Coll Cardiol 2008, 52(12):997-1003.

16. Savarese G, Musella F, D'Amore C, et al: Changes of natriuretic peptides predict hospital admissions in patients with chronic heart failure: a meta-analysis. JACC Heart Fail 2014, 2(2):148-158.

17. Savarese G, Hage C, Orsini N, et al: Reductions in N-terminal pro-brain natriuretic peptide levels are associated with lower mortality and heart failure hospitalization rates in patients with heart failure with mid-range and preserved 
ejection fraction. Circ Heart Fail 2016, 9(11).

18. Santaguida P L, Don-Wauchope A C, Oremus M, et al. BNP and NT-proBNP as prognostic markers in persons with acute decompensated heart failure: a systematic review.[J]. Heart Failure Reviews, 2014, 19(4):453-470.

19. Oremus M, Donwauchope A, Mckelvie R, et al. BNP and NT-proBNP as prognostic markers in persons with chronic stable heart failure.[J]. Heart Failure Reviews, 2014, 19(4):471-505.

20. Masson S, Latini R, Anand IS, et al. Direct comparison of B-type natriuretic peptide (BNP) and amino-terminal proBNP in a large population of patients with chronic and symptomatic heart failure: the Valsartan Heart Failure (Val-HeFT) data[J]. Clinical Chemistry, 2006, 52(8):1528.

21. Anand IS, Fisher LD, Chiang YT, et al. Changes in brain natriuretic peptide and norepinephrine over time and mortality and morbidity in the Valsartan Heart Failure Trial (Val-HeFT)[J]. Circulation, 2003, 107(9):1278-1283.

22. Paulo Bettencourt, Ana Azevedo, Joana Pimenta, et al: N-terminal-pro-brain natriuretic peptide predicts outcome after hospital discharge in heart failure patients[J]. Circulation, 2004, 110(15):2168-74.

23. Troughton R W, Frampton C M, Yandle T G, et al: Treatment of heart failure guided by plasma aminoterminal brain natriuretic peptide $(\mathrm{N}-\mathrm{BNP})$ concentrations $[\mathrm{J}]$. Lancet, 2000, 355(9210):1126-1130.

24. Shah M R,Califf $R$ M, Nohria A, et al. The STARBRITE Trial: A Randomized, Pilot Study of B-Type Natriuretic Peptide-Guided Therapy in Patients 
With Advanced Heart Failure[J]. journal of cardiac failure, 2011, 17(8):613-621.

25. Pfisterer M, Buser P, Rickli H, et al. BNP-guided vs symptom-guided heart failure therapy: The Trial of Intensified vs Standard Medical Therapy in Elderly Patients With Congestive Heart Failure (TIME-CHF) Randomized Trial[J]. JAMA, 2009, 301(4):383-92.

26. Eurlings L W M, Pol P E J V, Kok W E, et al. Management of chronic heart failure guided by individual N-terminal pro-B-type natriuretic peptide targets: Results of the PRIMA (Can PRo-brain-natriuretic peptide guided therapy of chronic heart failure IMprove heart fAilure morbidity and mortality?) [J]. Journal of the American College of Cardiology, 2010, 56(25):2090-100. 
Fig. 1. Flow diagram of included and excluded patients in the study.

Fig. 2. Distributions of $\log 10(\mathrm{NT}-$ proBNP) and $\log 10(\mathrm{NT}-$ proBNP) variability values.

Fig. 3. ROC curve of the Log10(NT-proBNP) variability and mean

Log10(NT-proBNP) as test variable and primary outcome.

Fig. 4. Kaplan-Meier curves comparing freedom from primary outcomes vs.

$\log 10(\mathrm{NT}-$ proBNP) variability in patients with CRS type 2.

Fig. 5. ROC curve of $\log 10(\mathrm{NT}$-proBNP) variability and mean Log10(NT-proBNP) as a test variable and secondary outcomes.

Fig. 6. Kaplan-Meier curves comparing freedom from Secondary outcomes vs. $\log 10(\mathrm{NT}-$ proBNP) variability in patients with CRS type 2.

Table 1. Baseline clinical characteristics according to NT-proBNP variability

\begin{tabular}{llll}
\hline Characteristics & $\begin{array}{l}\text { NT-proBNP variability } \\
<0.27(\mathrm{n}=67)\end{array}$ & $\begin{array}{l}\text { NT-proBNP variability } \\
\geq 0.27(\mathrm{n}=69)\end{array}$ \\
\hline Age (years) & $74.3 \pm 11.7$ & $75.9 \pm 9.9$ & 0.387 \\
Males, n (\%) & $36(52.2)$ & $31(44.9)$ & 0.394 \\
BMI $\left(\mathrm{kg} / \mathrm{m}^{2}\right)$ & $23.0 \pm 3.3$ & $23.2 \pm 3.2$ & 0.57 \\
Hypertension, n $(\%)$ & $51(73.9)$ & $55(79.7)$ & 0.42 \\
Cerebrovascular disease, $\mathrm{n}$ & $16(23.2)$ & $9(13.0)$ & 0.12 \\
$(\%)$ & & & 0.15 \\
Diabetes, $\mathrm{n}(\%)$ & $24(38.1)$ & $32(50 . .8)$ & 0.37 \\
COPD, $\mathrm{n}(\%)$ & $10(14.5)$ & $14(20.3)$ & 0.16 \\
AF, $\mathrm{n}(\%)$ & $21(33.3)$ & $29(42.0)$ & \\
\hline
\end{tabular}




\begin{tabular}{llll}
\hline NYHA $\geq$ III, n (\%) & $58(84.1)$ & $56(81.1)$ & 0.65 \\
LVEF (\%) & $52.5 \pm 10.7$ & $48.2 \pm 10.2$ & 0.02 \\
Creatinine (mg/dL) & $1.66 \pm 0.73$ & $1.60 \pm 0.77$ & 0.63 \\
eGFR (mL/min/1.73 m²) & $43.9 \pm 14.3$ & $44.6 \pm 12.6$ & 0.76 \\
SBP (mmHg) & $133.8 \pm 29.8$ & $133.5 \pm 23.0$ & 0.95 \\
DBP (mmHg) & $76.1 \pm 18.0$ & $75.7 \pm 14.6$ & 0.89 \\
Hb (g/L) & $123.8 \pm 20.9$ & $112.6 \pm 26.6$ & $<0.01$ \\
Alb (g/l) & $37.7 \pm 4.5$ & $35.8 \pm 4.7$ & $<0.02$ \\
ACEI or ARB, n (\%) & $56(81.2)$ & $55(79.7)$ & 0.83 \\
$\beta$-Blocker, n (\%) & $53(76.6)$ & $52(75.4)$ & 0.89 \\
spironolactone, n $(\%)$ & $54(78.2)$ & $50(72.5)$ & 0.43 \\
Loop diuretic, n (\%) & $60(86.9)$ & $57(85.1)$ & 0.42 \\
PCI, n (\%) & $24(34.7)$ & $27(39.1)$ & 0.59 \\
NT-proBNP (pg/mL) & $3678(1535,7650)$ & $3035(1515,6669)$ & 0.46 \\
Log10(NT-proBNP) & $3.48 \pm 0.61$ & $3.47 \pm 0.57$ & 0.94 \\
\hline
\end{tabular}

Notes: Continuous variables (age, BMI, LVEF, creatinine, eGFR, SBP, DBP, Hb, Alb, $\log 10(\mathrm{NT}$-proBNP), and NT-proBNP) are expressed as the mean $( \pm \mathrm{SD})$ or median (interquartile range), and categorical variables (men, hypertension, diabetes, cerebrovascular disease, COPD, liver disease, AF, cancer, NYHA $\geq$ III, ACEI or ARB, $\beta$-blockers, spironolactone, loop diuretic, and $\mathrm{PCI}$ ) are expressed as counts and percentages.

Abbreviations: ACEI, angiotensin-converting enzyme inhibitor; AF, atrial fibrillation; Alb, albumin; ARB, angiotensin II receptor blockers; BMI, body mass index; COPD, chronic obstructive pulmonary disease; DBP, diastolic blood pressure; eGFR, estimated glomerular filtration rate; $\mathrm{Hb}$, hemoglobin; LVEF, left ventricular ejection fraction; NT-proBNP, N-terminal pro-brain natriuretic peptide; NYHA, New York Heart Association (classification); PCI, percutaneous coronary intervention; SBP, systolic blood pressure.

Table 2. the number and incidence of primary outcome and second outcome 


\begin{tabular}{llll}
\hline primary outcome & $19(28.4 \%)$ & $33(47.8 \%)$ & 0.02 \\
Acute kidney disease & $6(8.9 \%)$ & $9(13.0 \%)$ & 0.45 \\
Nonfatal myocardial infarction & $7(10.4 \%)$ & $6(8.7 \%)$ & 0.75 \\
Cardiac death & $2(3.0 \%)$ & $11(15.9 \%)$ & 0.01 \\
Stroke & $2(3.0 \%)$ & $3(4.3 \%)$ & 1.0 \\
Dialysis & $4(6.0 \%)$ & $8(11.6 \%)$ & 0.25 \\
Secondary outcome & $24(35.8 \%)$ & $47(68.1 \%)$ & 0.001 \\
Repeated hospitalization for heart failure & $16(23.9 \%)$ & $29(42.0 \%)$ & 0.02 \\
All cause death & $8(11.9 \%)$ & $18(26.1 \%)$ & 0.03 \\
\hline
\end{tabular}

Table 3. Multivariate Cox regression analysis of the association between $\log _{10}($ NT-proBNP) variability and the primary outcomes. Model A: age, anemia, hypertension, diabetes mellitus, LVEF, hypoproteinemia, baseline eGFR and PCI. Model B: same covariates as model A plus $\log _{10}(\mathrm{NT}-$ proBNP) variability. Model C: same covariates as model A plus $\log _{10}($ NT-proBNP) variability as a categorical variable $(\geq 0.27$ and $<0.27)$.

\begin{tabular}{|c|c|c|c|c|c|c|c|c|}
\hline \multirow[b]{2}{*}{ Model A $\chi^{2}=25.337$} & \multirow[b]{2}{*}{$p=0.001$} & \multirow[b]{2}{*}{ B } & \multirow[b]{2}{*}{ S.E. } & \multirow[b]{2}{*}{ Wald } & \multirow[b]{2}{*}{ Sig. } & \multirow[b]{2}{*}{$\operatorname{Exp}(B)$} & \multicolumn{2}{|c|}{$95 \%$ CI for $\operatorname{Exp}(B)$} \\
\hline & & & & & & & Lower & Upper \\
\hline Age, years & & 0.016 & 0.016 & 0.949 & 0.330 & 1.016 & 0.984 & 1.048 \\
\hline Anemia (no, yes) & & -0.359 & 0.312 & 1.322 & 0.250 & 0.699 & 0.379 & 1.288 \\
\hline Hypertension (no, yes) & & -0.199 & 0.301 & 0.438 & 0.509 & 0.819 & 0.454 & 1.478 \\
\hline diabetes mellitus (no, yes) & & 0.335 & 0.301 & 1.240 & 0.266 & 1.397 & 0.775 & 2.519 \\
\hline LVEF (\%) & & 0.012 & 0.013 & 0.938 & 0.333 & 1.012 & 0.987 & 1.038 \\
\hline Hypoproteinemia (no, yes) & & 0.869 & 0.390 & 4.974 & 0.026 & 2.384 & 1.111 & 5.115 \\
\hline Baseline eGFR, $\mathrm{mL} / \mathrm{min} / 1.73 \mathrm{~m}^{2}$ & & -0.019 & 0.013 & 2.342 & 0.126 & 0.981 & 0.957 & 1.005 \\
\hline PCI (no, yes) & & -0.962 & 0.294 & 10.675 & 0.001 & 0.382 & 0.215 & 0.681 \\
\hline
\end{tabular}




\begin{tabular}{|c|c|c|c|c|c|c|c|c|}
\hline \multirow[b]{2}{*}{ Model B $\chi^{2}=29.217$} & \multirow[b]{2}{*}{$p=0.001$} & \multirow[b]{2}{*}{ B } & \multirow[b]{2}{*}{ S.E. } & \multirow[b]{2}{*}{ Wald } & \multirow[b]{2}{*}{ Sig. } & \multicolumn{3}{|c|}{$95 \%$ CI for $\operatorname{Exp}(B)$} \\
\hline & & & & & & $\operatorname{Exp}(B)$ & Lower & Upper \\
\hline $\log _{10}(\mathrm{NT}-$ proBNP) variability & & 0.660 & 0.320 & 4.262 & 0.039 & 1.935 & 1.034 & 3.622 \\
\hline Age, years & & 0.020 & 0.016 & 1.588 & 0.208 & 1.020 & 0.989 & 1.052 \\
\hline Anemia (no, yes) & & -0.359 & 0.315 & 1.292 & 0.255 & 0.699 & 0.377 & 1.298 \\
\hline Hypertension (no, yes) & & -0.172 & 0.301 & 0.328 & 0.568 & 0.842 & 0.467 & 1.518 \\
\hline diabetes mellitus (no, yes) & & 0.425 & 0.305 & 1.930 & 0.166 & 1.530 & 0.841 & 2.783 \\
\hline LVEF (\%) & & 0.017 & 0.013 & 1.759 & 0.185 & 1.017 & 0.992 & 1.044 \\
\hline Hypoproteinemia (no, yes) & & 0.667 & 0.400 & 2.773 & 0.096 & 1.948 & 0.889 & 4.270 \\
\hline Baseline eGFR, $\mathrm{mL} / \mathrm{min} / 1.73 \mathrm{~m}^{2}$ & & -0.019 & 0.013 & 2.399 & 0.126 & 0.980 & 0.955 & 1.005 \\
\hline \multirow[t]{2}{*}{ PCI (no, yes) } & & -0.924 & 0.299 & 9.575 & 0.002 & 0.392 & 0.225 & 0.711 \\
\hline & & & & & & & \multicolumn{2}{|c|}{$95 \%$ CI for $\operatorname{Exp}(B)$} \\
\hline Model C $\chi^{2}=27.057$ & $p=0.001$ & B & S.E. & Wald & Sig. & $\operatorname{Exp}(B)$ & Lower & Upper \\
\hline $\log _{10}(\mathrm{NT}-$ proBNP) variability $(\geqslant$ & & 1.243 & 0.440 & 7.982 & 0.01 & 3.467 & 1.463 & 8.215 \\
\hline \multicolumn{9}{|l|}{$0.27 \mathrm{OR}<0.27$ ) } \\
\hline Age, years & & 0.019 & 0.016 & 1.406 & 0.236 & 1.019 & 0.988 & 1.058 \\
\hline Anemia (no, yes) & & -0.355 & 0.315 & 1.269 & 0.265 & 0.702 & 0.378 & 1.306 \\
\hline Hypertension (no, yes) & & -0.183 & 0.301 & 0.369 & 0.543 & 0.833 & 0.464 & 1.508 \\
\hline diabetes mellitus (no, yes) & & 0.335 & 0.301 & 1.240 & 0.266 & 1.397 & 0.775 & 2.519 \\
\hline LVEF (\%) & & 0.018 & 0.013 & 1.823 & 0.177 & 1.018 & 0.992 & 1.044 \\
\hline Hypoproteinemia (no, yes) & & 0.709 & 0.401 & 3.136 & 0.077 & 2.033 & 0.927 & 4.459 \\
\hline Baseline eGFR, $\mathrm{mL} / \mathrm{min} / 1.73 \mathrm{~m}^{2}$ & & -0.020 & 0.014 & 2.432 & 0.119 & 0.980 & 0.956 & 1.007 \\
\hline PCI (no, yes) & & -0.940 & 0.294 & 9.955 & 0.002 & 0.391 & 0.219 & 0.701 \\
\hline
\end{tabular}

\section{Fig1}

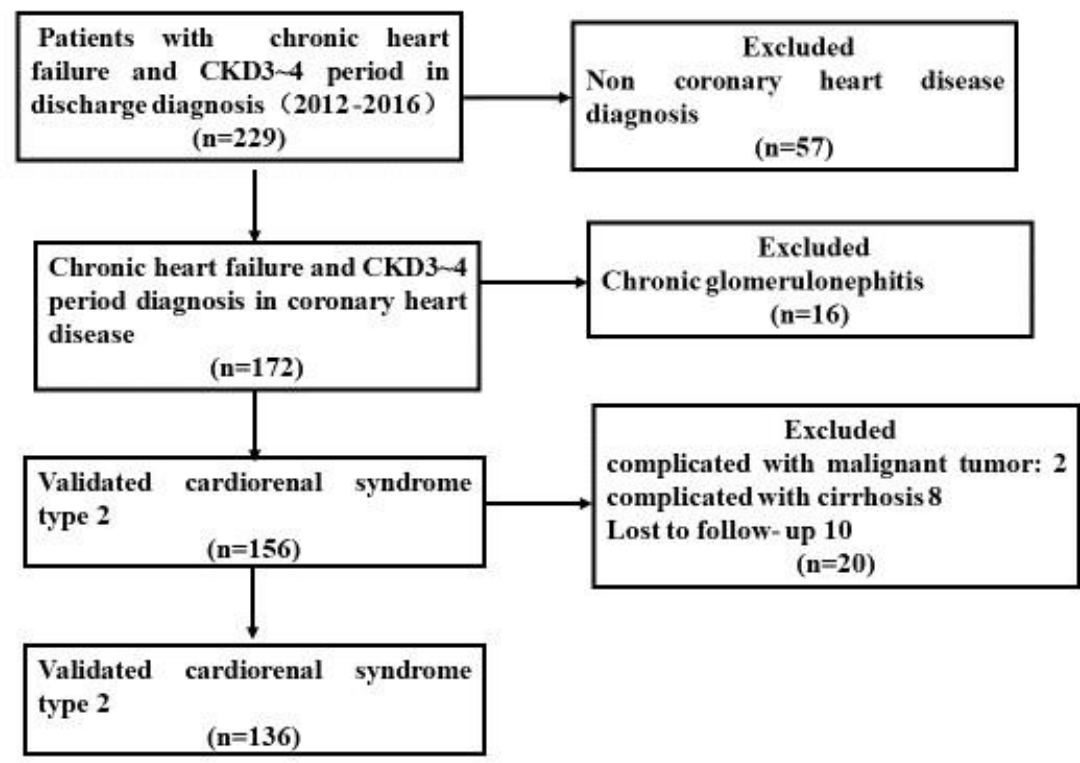


Fig2

A

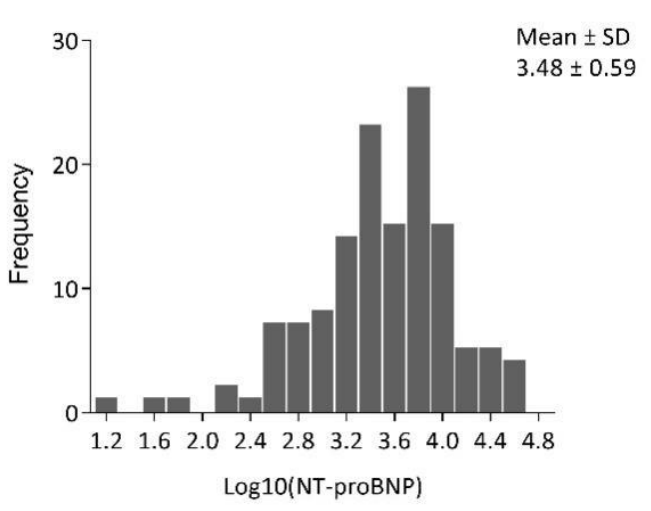

B

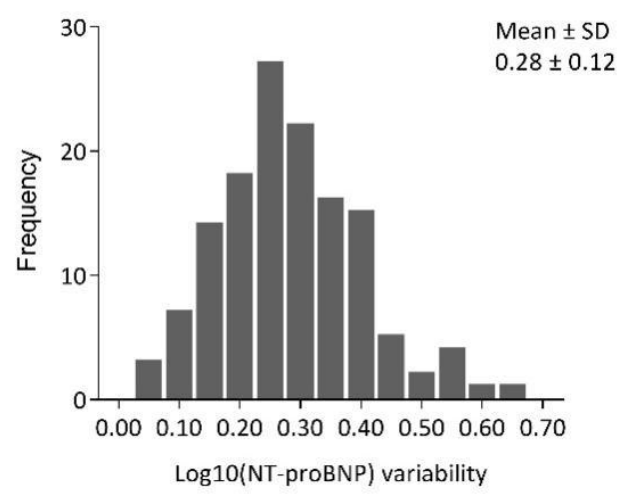

Fig3 


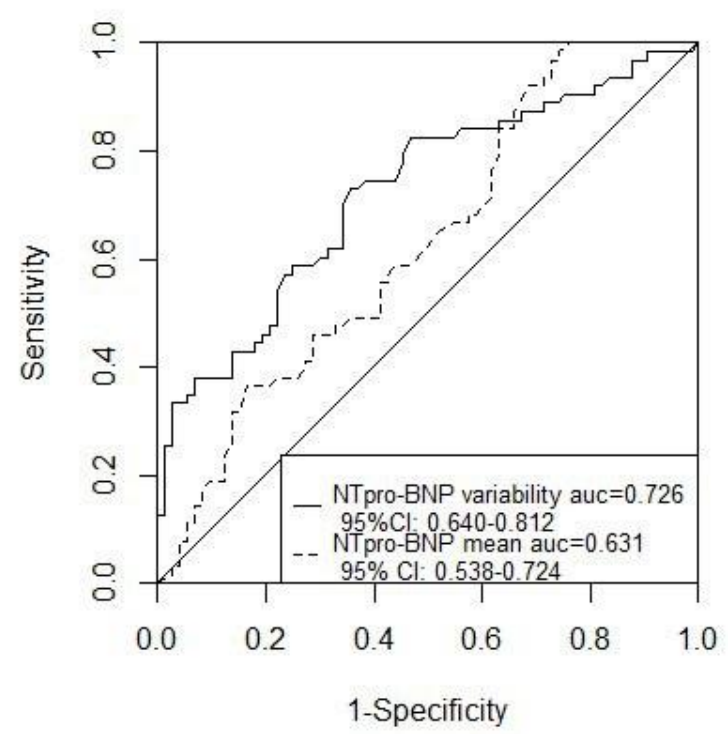

Fig4

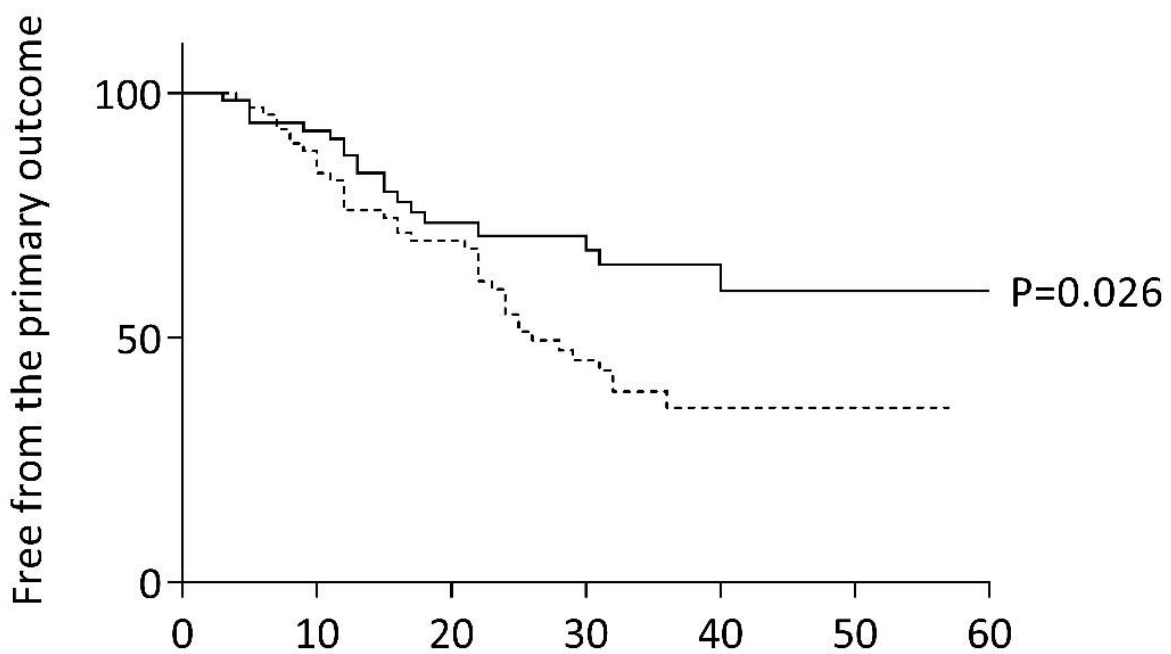

No. at Risk

Time(months)

Log10(NT-proBNP) variability

$\begin{array}{llllllll}0.2715 & 67 & 57 & 30 & 24 & 12 & 5 & 5\end{array}$

$\begin{array}{llllllll}\geq 0.2715 & 68 & 59 & 45 & 23 & 5 & 4 & 1\end{array}$

- $\log 10$ (NT-proBNP) variability $<0.2715$

-.-. $\log 10$ (NT-proBNP) variability $\geq 0.2715$ 
Fig5

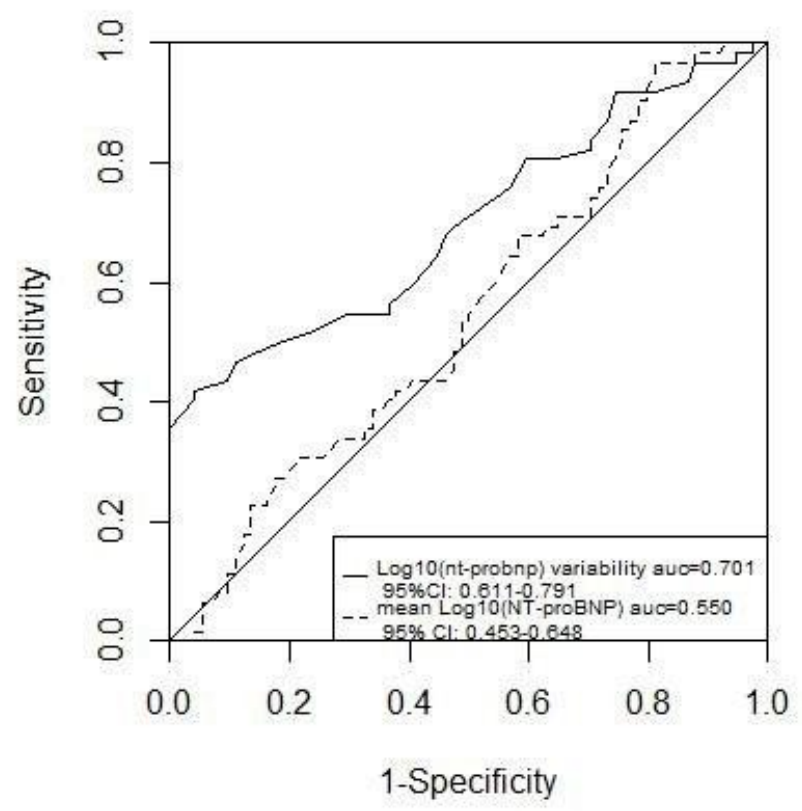

Fig6

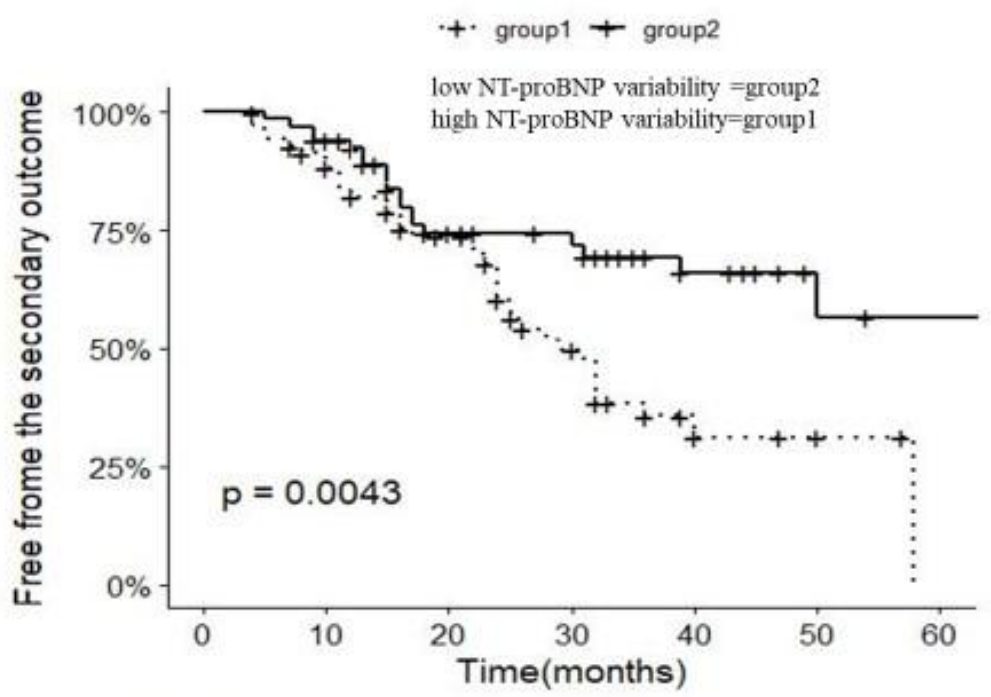

Number at risk

\begin{tabular}{|c|c|c|c|c|c|c|c|}
\hline group1 & 69 & 60 & 41 & 23 & 8 & 4 & 0 \\
\hline group2 & 67 & 61 & 36 & 30 & 18 & 7 & 5 \\
\hline & 0 & 10 & 20 & 30 & 40 & 50 & 60 \\
\hline
\end{tabular}


Figures

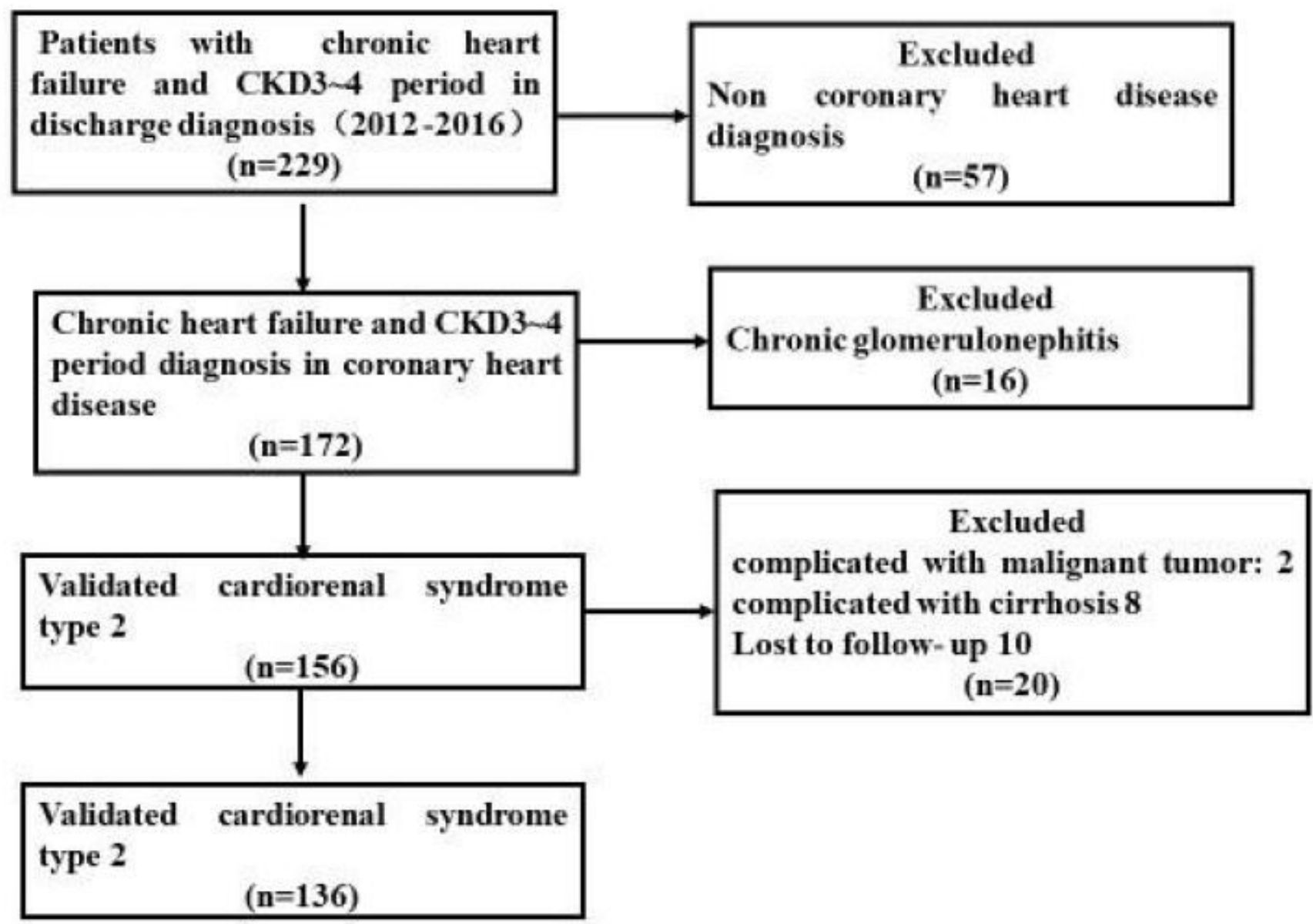

Figure 1

Flow diagram of included and excluded patients in the study. 
A

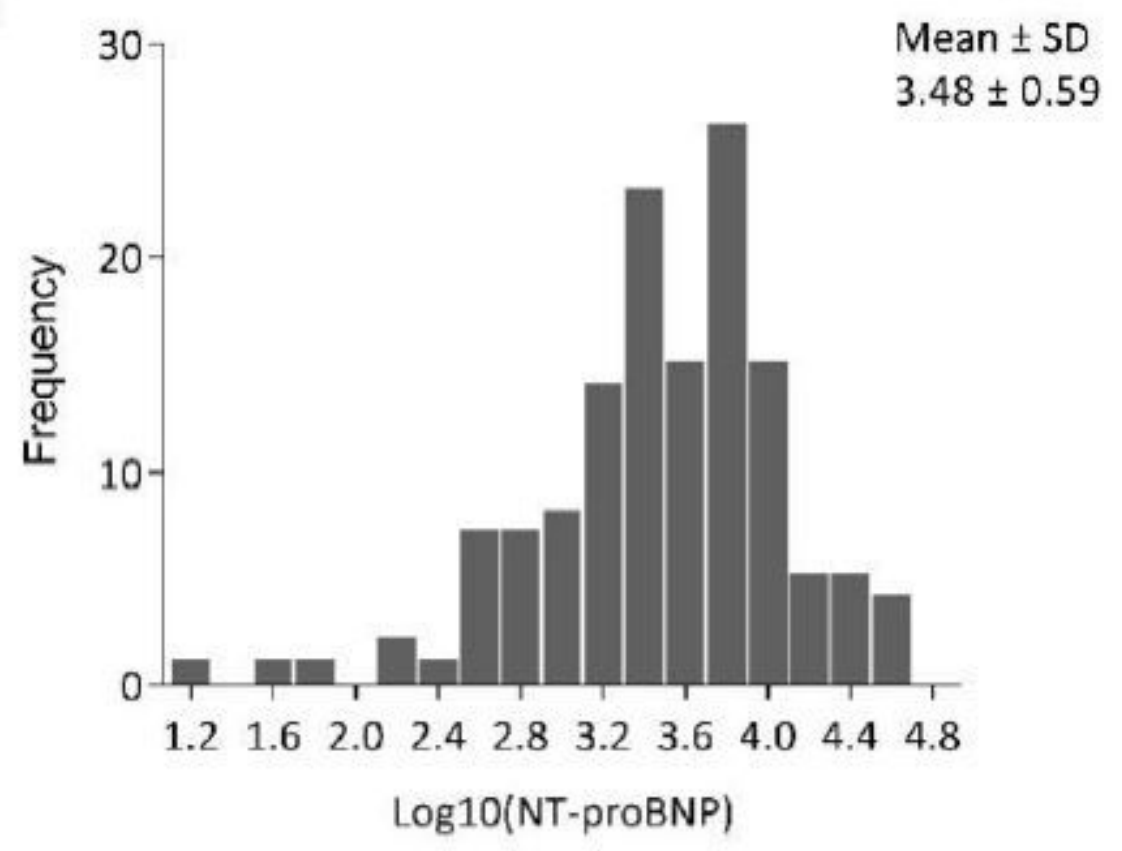

B

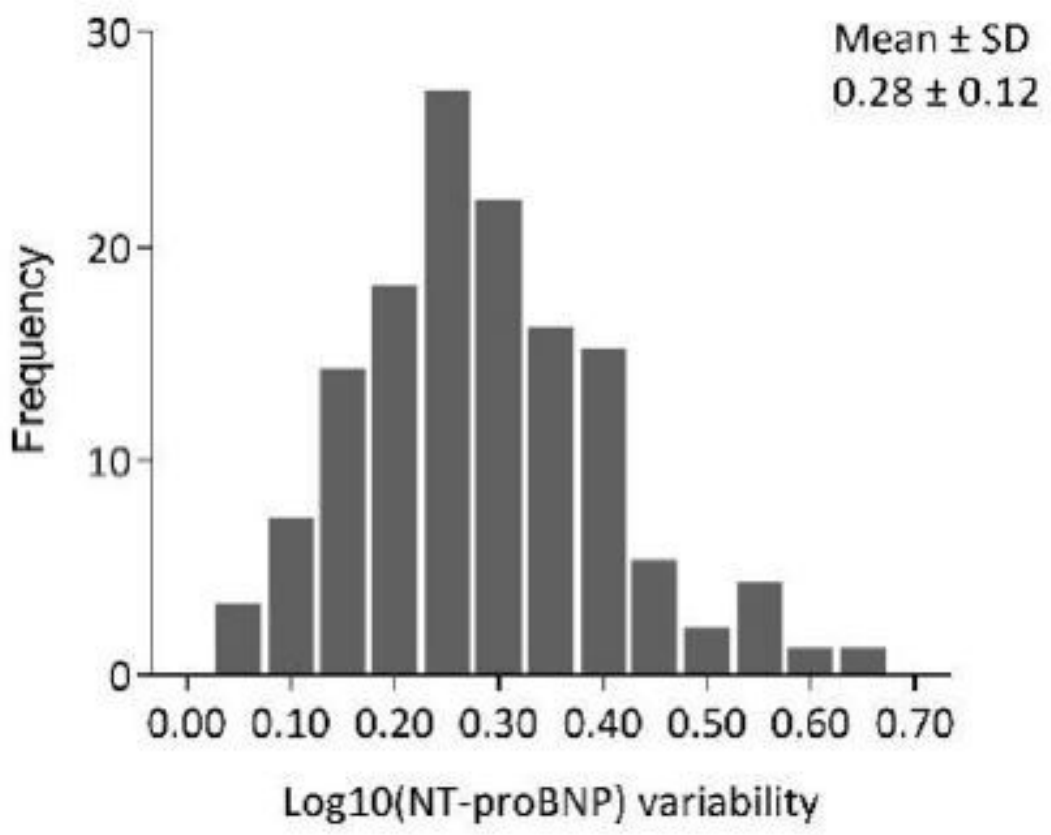

Figure 2

Distributions of log10(NT-proBNP) and log10(NT-proBNP) variability values. 


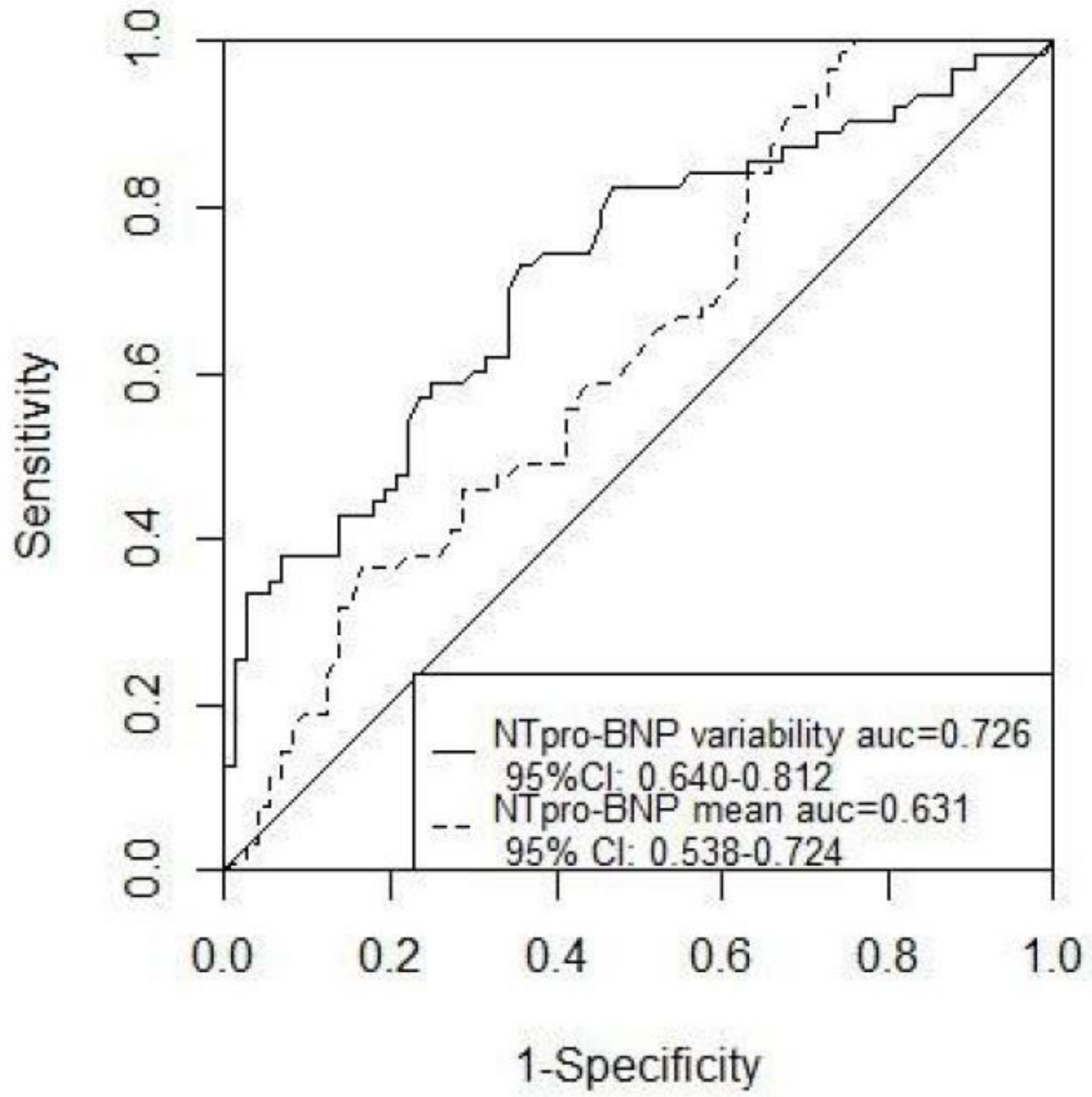

Figure 3

ROC curve of the Log10(NT-proBNP) variability and mean Log10(NT-proBNP) as test variable and primary outcome. 


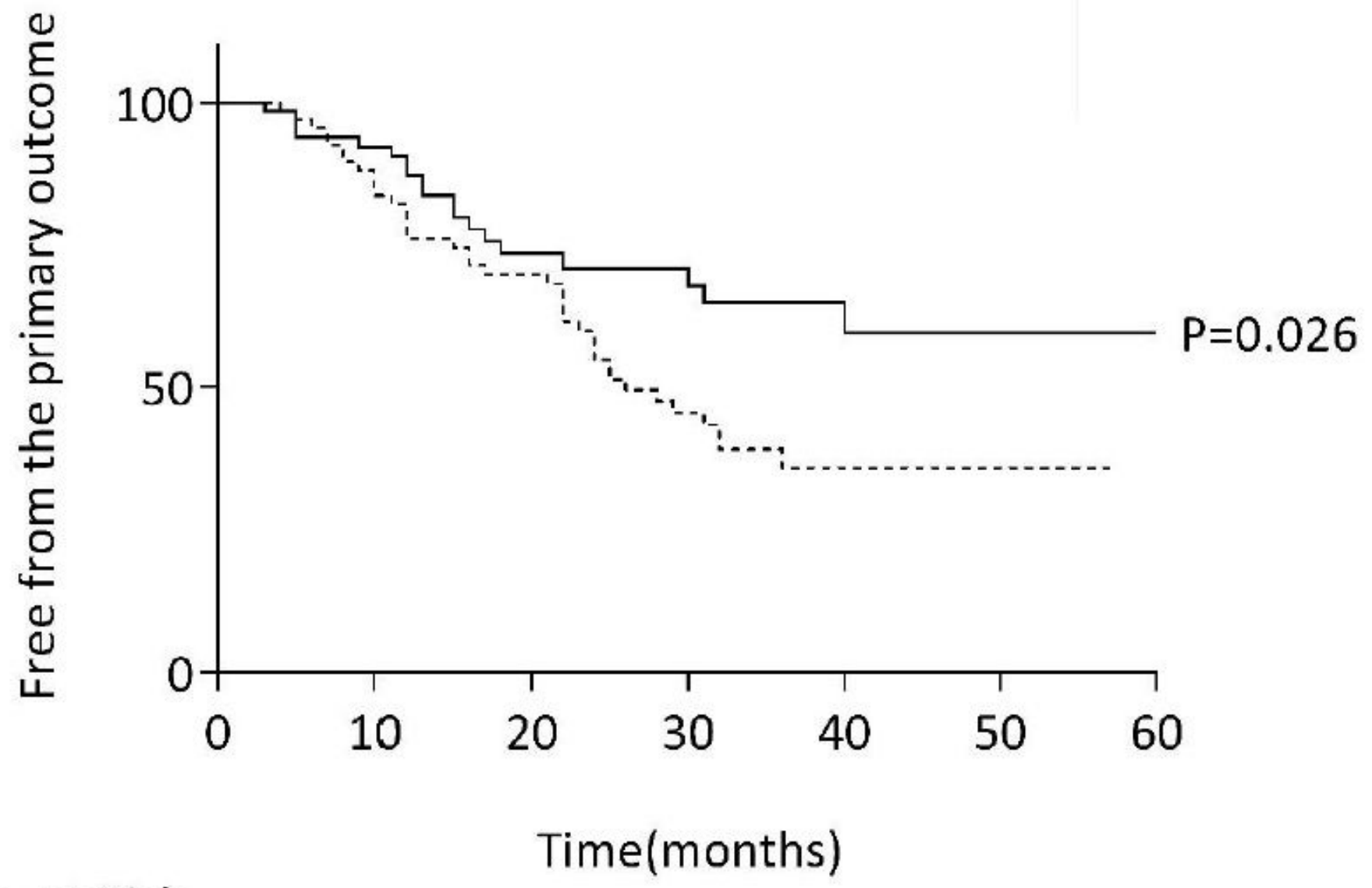

No. at Risk

Log10(NT-proBNP) variability

$\begin{array}{lllllcll}<0.2715 & 67 & 57 & 30 & 24 & 12 & 5 & 5 \\ \geq 0.2715 & 68 & 59 & 45 & 23 & 5 & 4 & 1\end{array}$

- $\log 10$ (NT-proBNP) variability $<0.2715$

-... $\log 10$ (NT-proBNP) variability $\geq 0.2715$

Figure 4

Kaplan-Meier curves comparing freedom from primary outcomes vs. log10(NT-proBNP) variability in patients with CRS type 2 . 


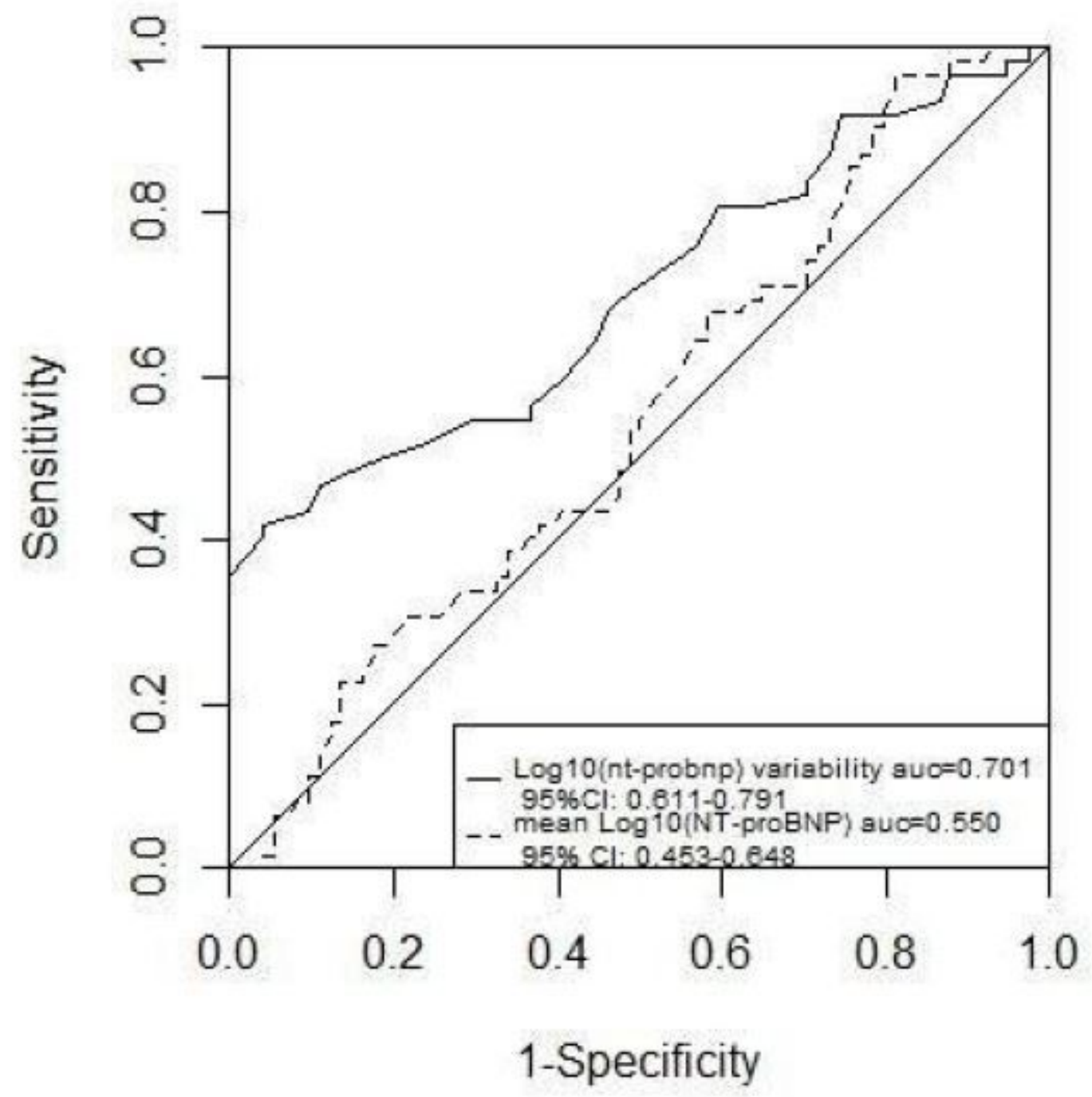

Figure 5

ROC curve of $\log 10($ NT-proBNP) variability and mean Log10(NT-proBNP) as a test variable and secondary outcomes. 


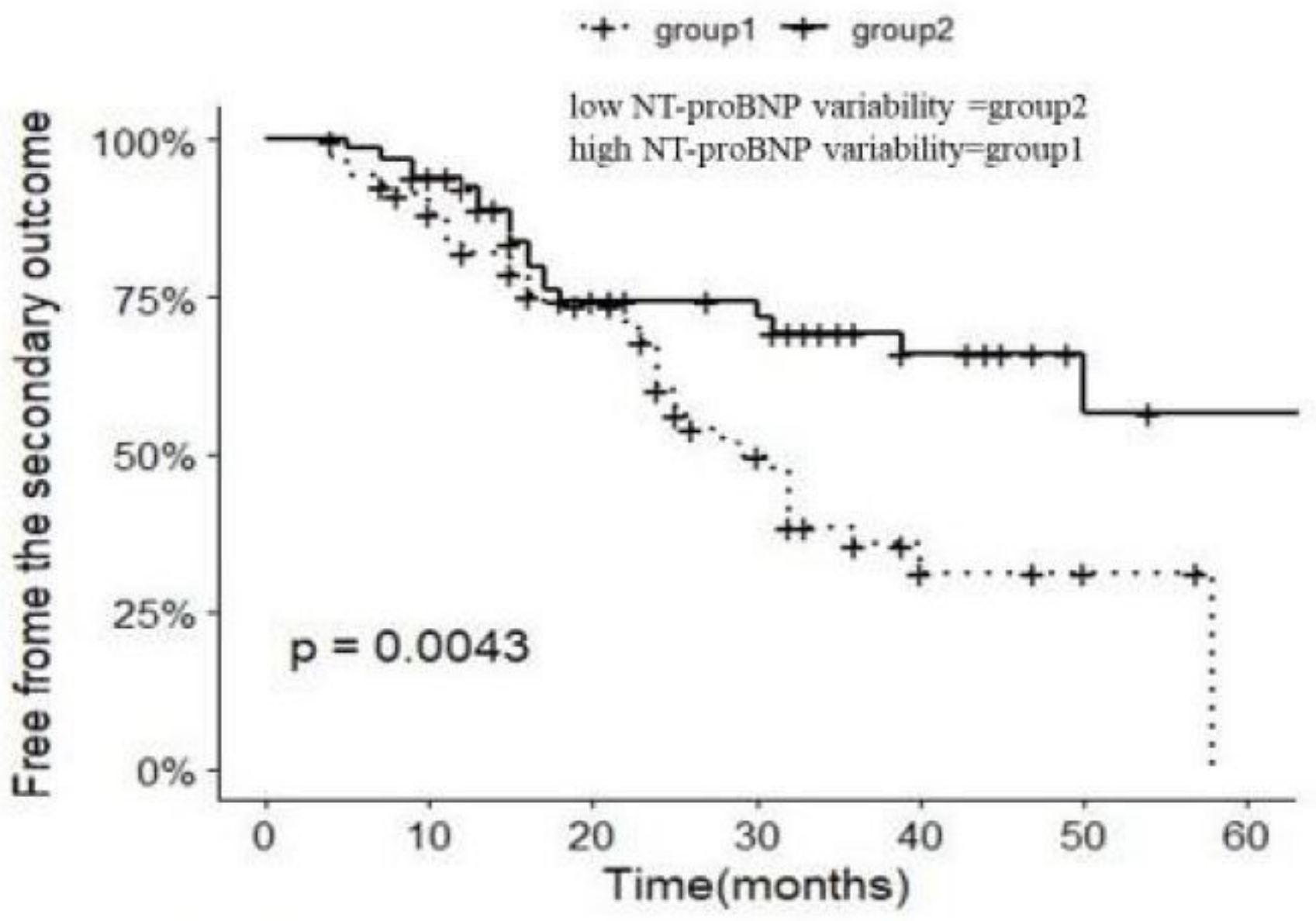

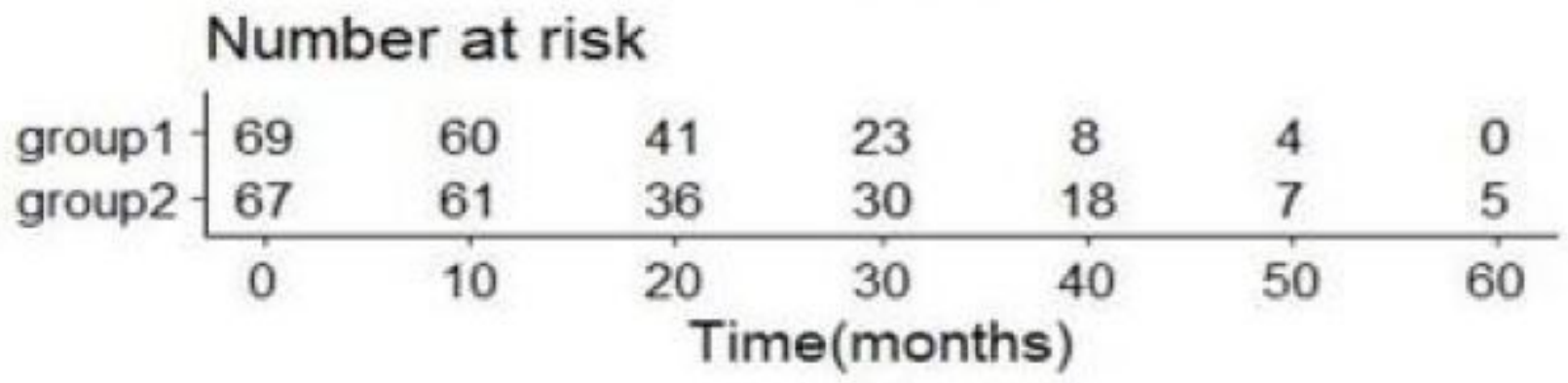

Figure 6

Kaplan-Meier curves comparing freedom from Secondary outcomes vs. $\log 10$ (NT-proBNP) variability in patients with CRS type 2 . 